\title{
LINC00958 and HOXC13-AS as key candidate biomarkers in head and neck squamous cell carcinoma by integrated bioinformatics analysis
}

\author{
Dan Xiong ${ }^{1,2,3}$, Wei Wu ${ }^{1}$, Lijuan Kan ${ }^{1}$, Dayang Chen ${ }^{1}$, Xiaowen Dou ${ }^{1}$, Xiang Ji ${ }^{1}$, Mengmeng Wang ${ }^{1}$, Zengyan Zong \\ , Jian Li ${ }^{\text {Corresp., } 2,3}$, Xiuming Zhang ${ }^{\text {Corresp. } 1}$ \\ ${ }^{1}$ Medical Laboratory of the Third affiliated hospital of ShenZhen university, Shenzhen, China \\ 2 Department of Otolaryngology, The First Affiliated Hospital, Sun Yat-sen University, Guangzhou, China \\ ${ }^{3}$ Guangzhou Key Laboratory of Otorhinolaryngology, Guangzhou, China \\ Corresponding Authors: Jian Li, Xiuming Zhang \\ Email address: lijianent@hotmail.com, zxm0760@163.com
}

Background: Head and neck squamous cell carcinoma (HNSCC) is a malignant tumor with a strong tendency for metastasis and recurrence. Finding effective biomarkers for the early diagnosis of HNSCC is critical for the early treatment and prognosis of patients.

Methods: RNA sequencing data including long non-coding RNAs (IncRNAs), messenger RNA (mRNAs) and microRNAs (miRNAs) of 141 HNSCC and 44 adjacent normal tissues were obtained from the TCGA. Differentially expressed genes were analyzed using the R package DESeq . GO terms and Kyoto Encyclopedia of Genes and Genomes $\square$ KEGG $\square$ pathway enrichment analyses were conducted. A competing endogenous RNAs $\square$ ceRNA $\square$ network was constructed. The most differentially expressed genes in the main ceRNA network were chosen for nasopharyngeal carcinoma (NPC) cell lines and NPEC2 Bmi-1 cell line verification. A receiver operating characteristic (ROC) curve was constructed for 141 specimens of HNSCC tissues from 44 control samples.

Results: In our study, 79 HNSCC-associated abnormally expressed IncRNAs , 86 abnormally expressed miRNAs and 324 abnormally expressed mRNAs were identified. The public microarray results showed that LINC00958 and HOXC13-AS expression levels were upregulated in HNSCC tissues compared with the matched adjacent normal tissues in this study $(p<0.0001)$. LINC00958 and HOXC13-AS expression levels in NPC cell lines were higher than those in the NPEC2 Bmi-1 cell line $(p<0.05)$. The results showed that the area under the ROC curve (AUC) of LINC00958 reached up to 0.906 at a cutoff value of 7.96, with a sensitivity and specificity of $80.85 \%$ and $90.91 \%$, respectively. The AUC of HOXC13-AS reached up to 0.898 at a cutoff value of 0.695 , with sensitivity and specificity values of $86.23 \%$ and $83.78 \%$, respectively.

Conclusion: The current study indicates that LINC00958 and HOXC13-AS are new candidate diagnostic biomarkers for HNSCC patients. 


\section{LINC00958 and HOXC13-AS as key}

2 candidate biomarkers in head and neck

3 squamous cell carcinoma by integrated ${ }_{4}$ bioinformatics analysis

5

6 Dan Xiong ${ }^{1}$, Wei Wu ${ }^{1}$, Lijuan $\mathrm{Kan}^{1}$, Dayang Chen ${ }^{1}$, Xiaowen Dou ${ }^{1}$, Xiang $\mathrm{Ji}^{1}$, Mengmeng Wang ${ }^{1}$, 7 Zengyan Zong ${ }^{1}$, Jian Li*2,3, Xiuming Zhang*1

$9{ }^{1}$ Medical Laboratory of the third affiliated hospital of Shenzhen university, Shenzhen, 518001, 10 China

11 2Department of Otolaryngology, The First Affiliated Hospital, Sun Yat-sen University, 12 Guangzhou, China

$13{ }^{3}$ Guangzhou Key Laboratory of Otorhinolaryngology, Guangzhou, China

14 *To whom correspondence may be addressed. Email: zxm0760@163.com or 15 lijianent@hotmail.com

Abstract

Background: Head and neck squamous cell carcinoma (HNSCC) is a malignant tumor with a strong tendency for metastasis and recurrence. Finding effective biomarkers for the early diagnosis of HNSCC is critical for the early treatment and prognosis of patients.

Methods: RNA sequencing data including long non-coding RNAs (lncRNAs), messenger RNA (mRNAs) and microRNAs (miRNAs) of 141 HNSCC and 44 adjacent normal tissues were obtained from the TCGA. Differentially expressed genes were analyzed using the R package DESeq. GO terms and Kyoto Encyclopedia of Genes and Genomes(KEGG) pathway enrichment analyses were conducted. A competing endogenous RNAs(ceRNA) network was constructed. The most differentially expressed genes in the main ceRNA network were chosen for nasopharyngeal carcinoma (NPC) cell lines and NPEC2 Bmi-1 cell line verification. A receiver operating 
characteristic (ROC) curve was constructed for 141 specimens of HNSCC tissues from 44 control samples.

Results: In our study, 79 HNSCC-associated abnormally expressed lncRNAs, 86 abnormally expressed miRNAs and 324 abnormally expressed mRNAs were identified. The public microarray results showed that LINC00958 and HOXC13-AS expression levels were upregulated in HNSCC tissues compared with the matched adjacent normal tissues in this study $(\mathrm{p}<0.0001)$. LINC00958 and HOXC13-AS expression levels in NPC cell lines were higher than those in the NPEC2 Bmi-1 cell line $(\mathrm{p}<0.05)$. The results showed that the area under the ROC curve (AUC) of LINC00958 reached up to 0.906 at a cutoff value of 7.96, with a sensitivity and specificity of $80.85 \%$ and $90.91 \%$, respectively. The AUC of HOXC13-AS reached up to 0.898 at a cutoff value of 0.695 , with sensitivity and specificity values of $86.23 \%$ and $83.78 \%$, respectively.

Conclusion: The current study indicates that LINC00958 and HOXC13-AS are new candidate diagnostic biomarkers for HNSCC patients.

Keywords: LINC00958; HOXC13-AS; biomarkers; HNSCC

\section{Introduction}

Head and neck squamous cell carcinoma (HNSCC) includes a group of tumors arising in the oral cavity, oropharynx, nasal cavity and paranasal sinuses, larynx and hypopharynx(Economopoulou et al. 2019). HNSCC was reported as the sixth most common type of malignancy in human(Wu et al. 2019). Estimately 50,0000 new cases are being diagnosed annually(Ghafouri-Fard et al. 2019). Despite the progress made in overall therapy in recent years, the five-year overall survival rate for HNSCC patients is only about 50\%(Cossu et al. 2019; Ren et al. 2016). The poor prognosis of HNSCC patients are mainly because of local invasions, treatment-resistance, recurrence, and metastasis(Ren et al. 2016; Yang et al. 2019).Patients with recurrence or metastasis after treatment often have a poor prognosis, which is the main reason for the failure of treatment and the poor survival rate of HNSCC patients. Therefore, screening biomarkers of HNSCC for early detection, the prediction of prognosis and the monitoring of recurrence is of great significance for the clinical diagnosis and treatment of HNSCC.

To improve the diagnosis and prognosis of HNSCC, novel effective and sensitive biomarkers are needed. lncRNAs are a kind of noncoding RNA with a transcript length of more than $200 \mathrm{bp}$. Their 
59 important regulatory role in tumorigenesis and metastasis has been a research focus in recent years.

60 Although the clinical significance of lncRNAs and their function and mechanism in cancer are still

61 unclear, they are involved in cancer progression and development beyond all doubt. Studies have

62 indicated that lncRNAs can serve as potential biomarkers for the early diagnosis and prognosis of

63 multiple cancers, including HNSCC(Guglas et al. 2017; Li et al. 2019a). Recent reports have

64 indicated that lncRNAs have multiple biological functions in HNSCC, including signaling

65 regulation, invasion, metastasis, and potential prognostic biomarkers(Xiong et al. 2019; Zhang et

66 al. 2019b). Qiu et al. found that knockdown of RHPN1-AS1 inhibited cell migration, invasion and

67 proliferation of HNSCC, indicating that RHPN1-AS1, acting as an oncogene, may be a potential

68 diagnostic and therapeutic target in HNSCC(Qiu et al. 2019). Jiang et al. suggested that

69 LINC00460 facilitated PRDX1 entry into the nucleus and promoted EMT in HNSCC cells.

70 LINC00460 and PRDX1 can serve as both promising candidate prognostic predictors and potential targets for cancer therapy for HNSCC(Jiang et al. 2019). The prognostic and diagnostic value of lncRNAs in HNSCC should not be underestimated, and their clinical significance will allow patients with HNSCC to receive more personalized treatment. In the present study, The Cancer Genome Atlas (TCGA) database was used to identify differentially expressed genes (lncRNAs, miRNA and mRNA) between HNSCC and adjacent normal tissues. Gene Ontology (GO) terms and Kyoto Encyclopedia of Genes and Genomes (KEGG) pathway enrichment analyses were conducted to further understand the molecular

functions of mRNAs. A ceRNA coexpression network of HNSCC was constructed to help clarify the functions of the noncoding RNAs in HNSCC. Nasopharyngeal carcinoma (NPC) is a type of HNSCC with a remarkable regional and ethnic specificity that can lead to serious health problems in south China and southeastern Asia compared with the Western world(Economopoulou et al. 2019). To screen candidate biomarkers from these differentially expressed genes, particularly those in the ceRNA network, we performed real-time PCR on NPC and NPEC2 Bmi-1 cells lines. Finally, we identified two lncRNAs, LINC00958 and HOXC13-AS, as new candidate biomarkers for HNSCC patients. 
90 Genome Atlas (TCGA, https://cancergenome.nih.gov/) database. Firstly, we excluded patients that

91 were firstly histologic diagnosed with non-HNSCC, Furthermore, patients with incomplete data 92 information such as clinicopathologic data, or the presence of another malignancy besides 93 HNSCC, or received chemotherapy or radiotherapy were excluded. Finally, a total of 141 HNSCC 94 and 44 normal control samples contained mRNA, miRNA and lncRNA data were analyzed in this study. The complete sample information is in Supplement table 1. Tables 1 to 3 show the tumor stage, origins, and institutions information of these samples. Figure 1 shows the flow chart for bioinformatics analysis performed in this study.

Cell culture

NPEC2 Bmi-1 cells grown in keratinocyte/serum-free (KSF) medium (Invitrogen) are immortalized nasopharyngeal epithelial cells induced by Bmi-1 as described previously(Gao et al. 2019). All human nasopharyngeal carcinoma cell lines (SUNE1, SUNE2, 6-10B, 5-8F, CNE2 and HONE1) were maintained in our laboratory and cultured in RPMI 1640 medium (GIBCO) supplemented with 5\% fetal bovine serum (FBS, GIBCO). All cells were cultured in a humidified $5 \% \mathrm{CO} 2$ incubator at $37^{\circ} \mathrm{C}$.

\section{Quantitative real-time PCR}

106 Total RNA was extracted from cultured cells using Trizol reagent (Invitrogen, Grand Island, NY) and reversely transcribed using a reverse transcriptase kit (Invitrogen) according to the manufacturer's instructions. The cDNA was then used as template in the real-time PCR using Power SYBR Green qPCR SuperMix-UDG (Invitrogen). The procedure of the real-time PCR reaction as follow: 1) $50^{\circ} \mathrm{C}$ for $2 \mathrm{~min}$; 2) pre-denaturation at $95^{\circ} \mathrm{C}$ for $10 \mathrm{~min}$; 3) denaturation at

$11195^{\circ} \mathrm{C}$ for $15 \mathrm{~s}$, annealing and extension at $60^{\circ} \mathrm{C}$ for $1 \mathrm{~min}, 40$ cycles. The real-time PCR reaction

112 was carried out on an ABI PRISM 7500 Sequence Detection System (Applied Biosystems, Foster

113 City, CA). All gene expression levels were normalized to that of the housekeeping gene GAPDH,

114 which was used as an internal standard. The forward primer for LINC00958 was 5'115 GCCTGGCACATTCAGTGGAGAG-3', and the reverse primer was

116 GTGGCGGCCTGAGCTTCTTC. The forward primer for HOXC13-AS was 5'117 CCTCAAGAAGACCAGCCGAAGTTG-3', and the reverse primer was 118 ATtGTtCAGAGCAAGCGGACTTCC. The forward primer for GAPDH was 5'119 CGAGGTCATAGTTCCTGTTGGTG-3', and the reverse primer was 120 CCCAATACGACCAAATCCGTT. 


\section{Data processing and differential expression analysis}

122 Samples were divided into tumor tissues vs. adjacent nontumor tissues to detect the differentially 123 expressed genes (DEGs) of lncRNAs, mRNAs and miRNAs. The raw RNA sequencing profile 124 was postprocessed and normalized by the TCGA RNASeqV2 system. No further normalization 125 was required. Ensemble gene IDs (ENSGs) were mapped to gene symbols and gene types (protein126 coding genes or noncoding genes) according to the annotation files in the GENCODE database 127 (version 22). The DEGs between the NPC and normal samples were analyzed using the R package 128 DESeq (http://www.bioconductor.org/packages/release/bioc/html/DESeq.html). A |log foldchange $(\mathrm{FC}) \mid \geq 2$ and a false discovery rate $(\mathrm{FDR})<0.01$ were set as the cut-off criteria.

\section{Functional and pathway enrichment analyses}

131 To further understand the biological processes, molecular functions and signaling pathways of the 132 protein-coding genes (mRNAs) among the differentially expressed RNAs, GO terms and KEGG 133 pathway enrichment analyses were performed with the help of the Database for Annotation, 134 Visualization and Integrated Discovery (DAVID, https://david.ncifcrf.gov/). Up- and down135 regulated mRNAs were analyzed. The thresholds were set as a $\mathrm{p}$ value $<0.05$ and a false discovery 136 rate $(\mathrm{FDR})<0.01$.

\section{7 ceRNA and PPI networks}

138 Based on the relationship among the lncRNAs, miRNAs and mRNAs, a lncRNA-miRNA-mRNA 139 ceRNA network was built. miRanda (http:/www.microrna.org/) and TargetScan

140 (http://www.targetscan.org/) were used to predict the miRNA-mRNA and miRNA-lncRNA 141 interactions. According to the theory that lncRNAs can regulate the activities of mRNAs by acting 142 as miRNA sponges, the mRNAs with negatively regulated lncRNAs and miRNAs were selected 143 to construct the lncRNA-miRNA-mRNA ceRNA network. Only the lncRNAs, miRNAs, and 144 mRNAs with an upregulated fold change $>1.5$ or a downregulated fold change $<0.50$ and a $p$ value

$145<0.05$ were retained. Afterward, in the context of the coexpressed genes, the PPI network was 146 constructed via STRING (Version 10.5) (https://string-db.org/).

\section{Statistical analysis}

148 Statistical analyses were performed using the statistical software package SPSS 16.0. Differences 149 among variables were analyzed by 2-tailed Student's t tests. Data are presented as the mean \pm SD 150 unless otherwise indicated. $p$ values $\leq 0.05$ were considered statistically significant. 
152 Results

153 Differentially expressed IncRNAs, microRNAs and mRNAs in HNSCC and adjacent normal 154 tissues from the TCGA database

155 In this study, 141 HNSCC patient tissues and 44 adjacent normal tissues were selected from the 156 TCGA database. Then, the lncRNA, miRNA and mRNA expression of $141 \mathrm{HNSCC}$ tissues and 15744 adjacent normal tissues was compared to identify the significantly different RNAs through 158 certain criteria (upregulated fold change $>1.5$ or downregulated fold change $<0.50$ and $\mathrm{p}<0.05$ ).

159 Then, 79 HNSCC-associated abnormally expressed lncRNAs (37 upregulated and 42 160 downregulated), 86 abnormally expressed miRNAs (36 upregulated and 50 downregulated) and 161324 abnormally expressed mRNAs (158 upregulated and 166 downregulated) were identified. 162 Figure 2A shows the top 15 upregulated lncRNAs and the top 15 downregulated lncRNAs. Figure 163 2B shows the top 15 upregulated miRNAs and the top 15 downregulated miRNAs, and Figure 2C 164 shows the top 15 upregulated mRNAs and the top 15 downregulated mRNAs.

165 Gene Ontology and pathway analyses in HNSCC and adjacent normal tissues from the 166 TCGA database

167 Finally, both the up- and downregulated mRNAs were further analyzed using the GO database 168 (http://www.geneontology.org). These differentially expressed genes represent a measure of 169 significance of a function. For instance, for the upregulated mRNAs, the most enriched GO term 170 was "signal transduction", and other significant GO terms included "extracellular matrix 171 organization," "cell adhesion," and "neutrophil degranulation". For the downregulated mRNAs, 172 the most enriched GO term was "neutrophil degranulation" (Figure 3A-B). These data provided a 173 definitive functional description of the genes differentially expressed in HNSCC. We next 174 subjected these differentially expressed genes to pathway analysis, where the most enriched 175 network corresponding to the upregulated transcripts was "human papillomavirus infection", and 176 the most enriched network corresponding to the downregulated transcripts was "metabolic 177 pathways" (Figure 3 C-D).

\section{8 ceRNA and PPI network construction}

179 To establish the lncRNA-miRNA-mRNA ceRNA network, lncRNAs and mRNAs targeted by 180 miRNAs were identified from the above data. A total of 38 miRNAs, 66 lncRNAs and 102 mRNAs 181 were included in the ceRNA network (Figure 4). Seventy-two upregulated and 19 downregulated 182 mRNAs were included in the PPI network (Figure 5). Proteins with high degrees were classified 
183 as hub proteins. The most significant hub proteins were HSP90AA1, CD44, TOP2A and ITGAV.

184 LINC00958 and HOXC13-AS expression levels in tumors and cells.

185 The long noncoding RNA LINC00958 has been reported to facilitate cell proliferation and

186 migration in oral squamous cell carcinoma(Wang et al. 2019). HOXC13-AS was confirmed to 187 positively affect cell proliferation and invasion in nasopharyngeal carcinoma(Dai et al. 2019). Our

188 results showed that LINC00958 and HOXC13-AS expression levels were upregulated in HNSCC

189 tissues compared with that in the matched adjacent normal tissues in this study $(\mathrm{p}<0.0001$, Figure

190 6A-B). To validate the expression levels of LINC00958 and HOXC13-AS identified through our

191 bioinformatics analyses, real-time PCR was performed to detect the expression levels of

192 LINC00958 and HOXC13-AS in NPC cell lines and in the NPEC2 Bmi-1 cell line. LINC00958

193 and HOXC13-AS expression levels in the NPEC2 Bmi-1 cell line were lower than those in the

194 NPC cell lines $(\mathrm{p}<0.0001$, Figure 6C-D). ROC curve was constructed for 141 specimens of

195 HNSCC tissues from 44 control samples. The results showed that the area under the ROC curve

196 (AUC) of LINC00958 reached up to 0.906 (95\% confidence interval $(\mathrm{CI})=0.863-0.949 ; \mathrm{p}<0.001$,

197 Figure 6E). The optimal cutoff value was 7.96, with a sensitivity and specificity of $80.85 \%$ and

$19890.91 \%$, respectively. The results showed that the AUC of HOXC13-AS reached up to 0.898 (95\%

199 confidence interval $(\mathrm{CI})=0.834-0.962 ; \mathrm{p}<0.001$, Figure $6 \mathrm{~F})$. The optimal cutoff value of HOXC13-

200 AS expression was 0.695 , with sensitivity and specificity values of $86.23 \%$ and $83.78 \%$,

201 respectively.

202 Discussion

203 The TCGA is a comprehensive bioinformatics database that contains information on various 204 malignant tumors. Based on the TCGA bioinformatics analysis, many studies have been carried 205 out to improve our understanding of the pathological process of multiple cancers. Through an 206 integrative analysis of GEO and TCGA data, Zhang et al. suggested that three lncRNAs, UCA1, 207 HOTTIP, and HMGA1P4, may contribute to the development of gastric cancer and may be related 208 to the prognosis of gastric cancer patients(Zhang et al. 2019a). Wang et al. used bioinformatics 209 and showed that TRMT12 might involve in the progression and metastasis of HNSCC, and could 210 be served as an independent biomarker of poor prognosis in HNSCC(Wang et al. 2019). Dai et al. 211 found that HOXC10 facilitated WNT-dependent epithelial-mesenchymal transition (EMT) and 212 might be a potential prognostic biomarker and a therapeutic target in oral squamous cell carcinoma 213 (OSCC)(Dai et al. 2019). In our study, the AUC of LINC00958 reached up to 0.906. The optimal 
214 cutoff value was 7.96, with a sensitivity and specificity of $80.85 \%$ and $90.91 \%$, respectively. The 215 results showed that the AUC of HOXC13-AS reached up to 0.897. The optimal cutoff value of 216 HOXC13-AS expression was 0.695 , with sensitivity and specificity values of $86.23 \%$ and $83.78 \%$, 217 respectively._Finally, we identified two lncRNAs, LINC00958 and HOXC13-AS, as new candidate 218 biomarkers for HNSCC patients by bioinformatics analysis.

219 Several studies have suggested that LINC00958 and HOXC13-AS are significantly associated 220 with tumor progression, migration, and invasion. A recent report showed that LINC00958 221 regulated the miR-627-5p/YBX2 axis and facilitated cell proliferation and migration in oral 222 squamous cell carcinoma(Chen et al. 2019a). Wen et al. reported that silencing LINC00958 prevented tumor initiation by acting as a sponge of microRNA-330-5p to downregulate PAX8 in pancreatic cancer(Chen et al. 2019b). Seitz et al. showed that LINC00958 was significantly 225 upregulated in bladder cancer and exhibited phenotypic functions that showed carcinogenic characteristics(Seitz et al. 2017). A recent study reported that HOXC13-AS was obviously upregulated in breast cancers and promoted cell proliferation by regulating the miR-497-5p/PTEN axis(Li et al. 2019b). HOXC13-AS positively affects cell proliferation and invasion by modulating the miR-383-3p/HMGA2 axis in NPC(Gao et al. 2019). In our study, there was an obvious increase in LINC00958 and HOXC13-AS expression at the mRNA level between HNSCC and adjacent tissues. Next, the exact function and mechanism of LINC00958 and HOXC13-AS in HNSCC were further explored, including proliferation, invasion and metastasis.

233 The differentiation and progression of HNSCC cells may be closely related to the regulation of 234 certain genes. In our study, GO and pathway enrichment analyses revealed that the upregulated 235 factors are mainly involved in 'extracellular matrix organization', 'human papillomavirus 236 infection', 'focal adhesion', 'ECM-receptor interaction', 'PI3K-Akt signaling pathway', and 237 'pathway cancer', while the downregulated factors are associated with 'muscle filament sliding', 238 'cornification', 'metabolic pathways', 'cardiac muscle contraction' and 'fatty acid degradation'.

239 Extracellular matrix organization and cell adhesion play an important role in the invasion and 240 metastasis of HNSCC cells.

241 Although this study has demonstrated that LINC00958 and HOXC13-AS are useful in clinical 242 discrimination of HNSCC and adjacent tissues, several challenges remain. In the TCGA base 243 squamous cell carcinoma are arising from different origins. Then the LINC00958 and HOXC13244 AS expression value in different origins of HNSCC were compared using the Kruskal-Wallis Test 
245 for origin with a frequency of 10 or more. The results were showed in Supplement table 2. At $p$ 2460.05 significance level, we concluded that there is no difference between different origins of 247 squamous cell carcinoma. Supplement Figure 1 and Figure 2 showed LINC00958 and HOXC13248 AS expression value in different origins respectively. Another challenge is to obtain more clinical 249 samples validating results, especially those from nasopharynx site.

250 In conclusion, our study revealed differentially expressed lncRNAs, microRNAs and mRNAs 251 between nasopharyngeal carcinoma and adjacent normal tissues from the TCGA database. 252 Different Gene Ontology terms, pathways, and ceRNA and PPI networks were obtained. We 253 identified two lncRNAs, LINC00958 and HOXC13-AS, as new candidate biomarkers for NPC 254 patients by bioinformatics analysis. These results indicate that the transcript levels of LINC00958 255 and HOXC13-AS may be suitable biomarkers of NPC.

\section{Competing interests}

257 The authors declare that they have no competing interests.

258 Acknowledgement

259 This study was supported by the National Natural Science Foundation of China (Grant 260 NO.81772921, 81502344). A grant from the Science and Technology Planning Project of Shenzhen City of China (NO. JCYJ20180306172209668). The discipline construction ability promotion project of Shenzhen health and population family planning commission (NO. SZXJ2017018). Sanming Project of Medicine in Shenzhen (NO. SZSM201601062). The Natural Science Foundation of Guangdong Province (NO.2016A030313257). The Science and Technology Planning Project of Guangdong Province ( NO.2014A020212141).

\section{References}

Chen F, Liu M, Yu Y, Sun Y, Li J, Hu W, Wang X, and Tong D. 2019a. LINC00958 regulated miR-627-5p/YBX2 axis to facilitate cell proliferation and migration in oral squamous cell carcinoma. Cancer biology \& therapy:1-11.

272 Chen S, Chen J-Z, Zhang J-Q, Chen H-X, Qiu F-N, Yan M-L, Tian Y-F, Peng C-H, Shen B-Y, 273 and Chen Y-L. 2019b. Silencing of long noncoding RNA LINC00958 prevents tumor initiation of 
274 pancreatic cancer by acting as a sponge of microRNA-330-5p to down-regulate PAX8. Cancer 275 letters 446:49-61.

276 Cossu AM, Mosca L, Zappavigna S, Misso G, Bocchetti M, De Micco F, Quagliuolo L, Porcelli

277 M, Caraglia M, and Boccellino M. 2019. Long Non-coding RNAs as Important Biomarkers in

278 Laryngeal Cancer and Other Head and Neck Tumours. International journal of molecular sciences $27920: 3444$.

280 Dai B-W, Yang Z-M, Deng P, Chen Y-R, He Z-J, Yang X, Zhang S, Wu H-J, and Ren Z-H. 2019.

281 HOXC10 promotes migration and invasion via the WNT-EMT signaling pathway in oral 282 squamous cell carcinoma. Journal of Cancer 10:4540.

283 Economopoulou P, Van Gestel D, Kotsantis I, and Psyrri A. 2019. Diagnostic tumor markers in 284 head and neck squamous cell carcinoma (HNSCC) in the clinical setting. Frontiers in oncology $2859: 827$.

286 Gao C, Lu W, Lou W, Wang L, and Xu Q. 2019. Long noncoding RNA HOXC13-AS positively 287 affects cell proliferation and invasion in nasopharyngeal carcinoma via modulating 288 miR-383-3p/HMGA2 axis. Journal of Cellular Physiology 234:12809-12820.

289 Ghafouri-Fard S, Mohammad-Rahimi H, Jazaeri M, and Taheri M. 2019. Expression and function 290 of long non-coding RNAs in head and neck squamous cell carcinoma. Experimental and 291 Molecular Pathology:104353.

292 Guglas K, Bogaczyńska M, Kolenda T, Ryś M, Teresiak A, Bliźniak R, Łasińska I, Mackiewicz 293 J, and Lamperska K. 2017. IncRNA in HNSCC: challenges and potential. Contemporary Oncology $294 \quad 21: 259$.

295 Jiang Y, Cao W, Wu K, Qin X, Wang X, Li Y, Yu B, Zhang Z, Wang X, and Yan M. 2019. 296 LncRNA LINC00460 promotes EMT in head and neck squamous cell carcinoma by facilitating 297 peroxiredoxin-1 into the nucleus. Journal of Experimental \& Clinical Cancer Research 38:365.

$298 \mathrm{Li} \mathrm{J}, \mathrm{Li} \mathrm{Y}, \mathrm{Wu}$ X, and Li Y. 2019a. Identification and validation of potential long non-coding RNA 299 biomarkers in predicting survival of patients with head and neck squamous cell carcinoma. 300 Oncology letters 17:5642-5652.

301 Li X, Wang Q, Rui Y, Zhang C, Wang W, Gu J, Tang J, and Ding Y. 2019b. HOXC13-AS 302 promotes breast cancer cell growth through regulating miR-497-5p/PTEN axis. Journal of Cellular 303 Physiology. 
304 Qiu X, Lei Z, Wang Z, Xu Y, Liu C, Li P, Wu H, and Gong Z. 2019. Knockdown of LncRNA

305 RHPN1-AS1 inhibits cell migration, invasion and proliferation in head and neck squamous cell 306 carcinoma. Journal of Cancer 10:4000.

307 Ren Z-H, Lin C-Z, Cao W, Yang R, Lu W, Liu Z-Q, Chen Y-M, Yang X, Tian Z, and Wang L-Z. 308 2016. CD73 is associated with poor prognosis in HNSCC. Oncotarget 7:61690.

309 Seitz AK, Christensen LL, Christensen E, Faarkrog K, Ostenfeld MS, Hedegaard J, Nordentoft I,

310 Nielsen MM, Palmfeldt J, and Thomson M. 2017. Profiling of long non-coding RNAs identifies

311 LINC00958 and LINC01296 as candidate oncogenes in bladder cancer. Scientific reports 7:395.

312 Wang K, Zheng M, and Ren Y. 2019. Overexpression of TRMT12 may independently predict poor

313 overall survival in patients with head and neck squamous cell carcinoma. OncoTargets and therapy $314 \quad 12: 7269$.

$315 \mathrm{Wu} \mathrm{H}, \mathrm{Yu} \mathrm{DH}, \mathrm{Wu} \mathrm{MH}$, and Huang T. 2019. Long non-coding RNA LOC541471: A novel

316 prognostic biomarker for head and neck squamous cell carcinoma. Oncol Lett 17:2457-2464.

$317 \quad 10.3892 / \mathrm{ol} .2018 .9831$

318 Xiong H-G, Li H, Xiao Y, Yang Q-C, Yang L-L, Chen L, Bu L-L, Zhang W-F, Zhang J-L, and

319 Sun Z-J. 2019. Long noncoding RNA MYOSLID promotes invasion and metastasis by modulating

320 the partial epithelial-mesenchymal transition program in head and neck squamous cell carcinoma.

321 Journal of Experimental \& Clinical Cancer Research 38:278.

322 Yang CX, Sedhom W, Song J, and Lu S-L. 2019. The role of MicroRNAs in recurrence and 323 metastasis of head and neck squamous cell carcinoma. Cancers 11:395.

324 Zhang X, Zhang W, Jiang Y, Liu K, Ran L, and Song F. 2019a. Identification of functional 325 IncRNAs in gastric cancer by integrative analysis of GEO and TCGA data. Journal of cellular 326 biochemistry.

327 Zhang ZL, Zhao LJ, Xu L, Chai L, Wang F, Xu YP, Zhou SH, and Fu Y. 2019b. Transcriptomic 328 model-based lncRNAs and mRNAs serve as independent prognostic indicators in head and neck 329 squamous cell carcinoma. Oncology letters 17:5536-5544.

Figure legends 
335 Figure 1 The flow chart for bioinformatics analysis in this study.

336 Figure 2 Cluster analysis of 15 up- and down-regulated differentially expressed IncRNAs,

337

338

339

340

341

342

343

344

345

346

347

348

349

350

351

352

353

354

355

356

357

358

359

360

361

362

363

364

365

\section{microRNA and mRNAs in HNSCC tissues and adjacent normal tissues from TCGA}

database. Hierarchical clustering analysis indicated that 15 up- and down-regulated differentially expressed lncRNAs (A), microRNA (B) and mRNAs (C) were differentially expressed between HNSCC patients and healthy controls, Respectively. The red and the green shades represent the expression levels above and below the relative expression among all samples.

Figure 3 The 20 key Gene Ontology (GO) terms and KEGG pathway enrichment of differentially expressed intersection mRNAs for HNSCC groups versus adjacent normal controls.

A. The 20 Key gene ontology in up-regulated differentially expressed genes, the bar plot shows the enrichment scores of the significant enrichment GO terms.

B. The 20 key gene ontology in down-regulated, differentially expressed genes, the bar plot shows the enrichment scores of the significant enrichment GO terms.

C. The 20 key pathways in up-regulated differentially expressed genes, the bar plot shows the enrichment scores of the significant enrichment pathways

D. The 20 key pathways in down-regulated differentially expressed genes.the bar plot shows the enrichment scores of the significant enrichment pathways.

Figure 4 The ceRNA network of IncRNA-miRNA-mRNA in HNSCC. The red represents the upregulated, and the green represents the downregulated. Diamonds represent miRNAs, balls represent mRNAs, and triangles represent lncRNAs.

Figure 5 PPI network of 72 upregulated and 19 downregulated DEGs. Nodes represent proteins and edges represent interactions between two proteins. Red nodes represent upregulated DEGs and green nodesrepresent downregulated DEGs.

Figure 6 LINC00958 and HOXC13-AS expression levels in tumors and cells.

A-B. Enhanced expression of LINC00958 and HOXC13-AS expression levels in HNSCC tissues compared with adjacent normal tissues $(\mathrm{n}=44, \mathrm{~T}=141, \mathrm{p}<0.0001)$.

C-D. LINC00958 and HOXC13-AS expression levels in NPC cell lines were higher than that in NPEC2 Bmi-1 which was immortalized nasopharyngeal epithelial cells induced by Bmi-1. E-F. The ROC curve of LINC00958 and HOXC13-AS between HNSCC tissues and controls. 
Figure 1

Figure 1 The flow chart for bioinformatics analysis in this study.

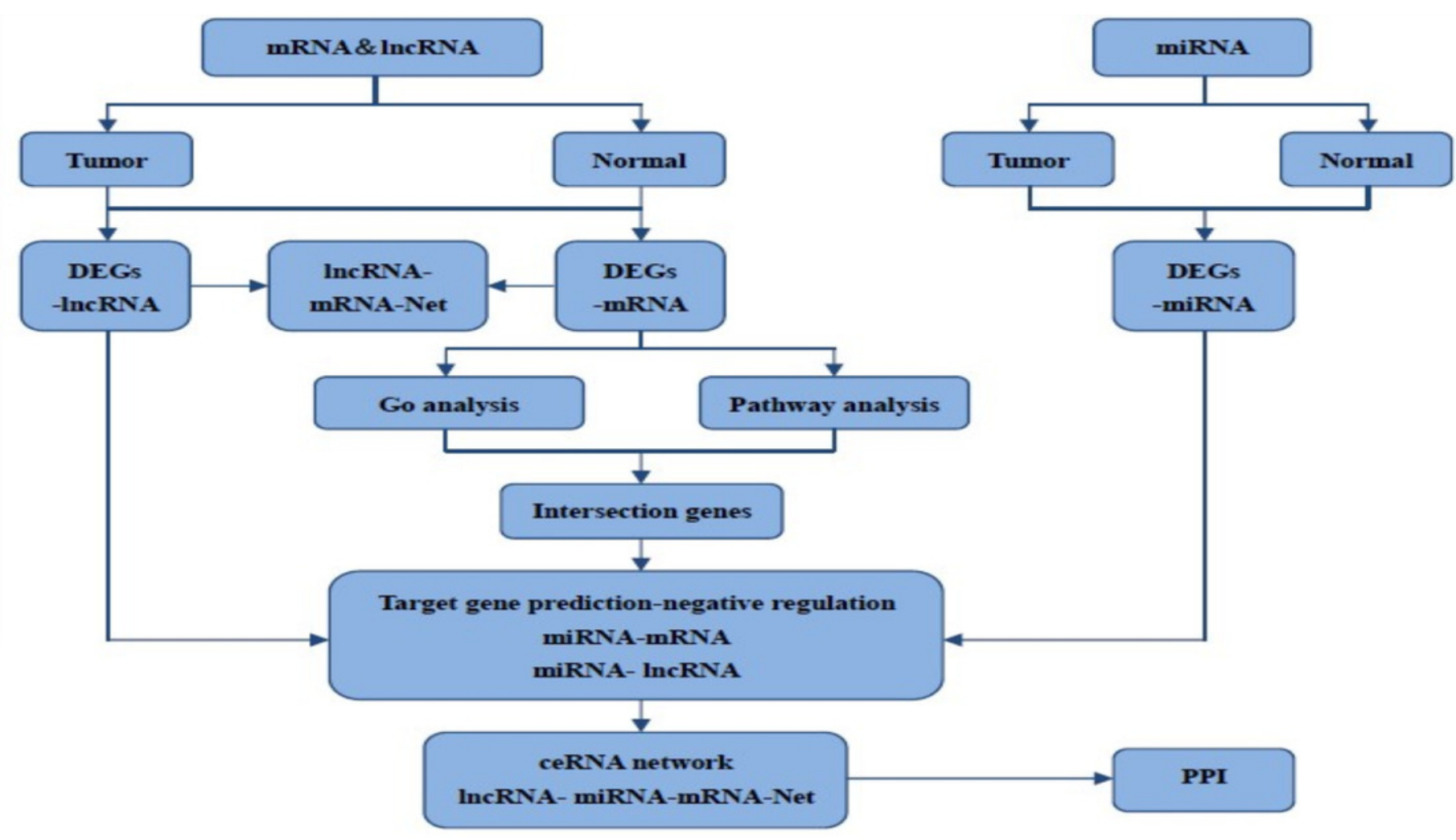




\section{Figure 2}

Figure 2 Cluster analysis of 15 up- and down-regulated differentially expressed IncRNAs, microRNA and mRNAs in HNSCC tissues and adjacent normal tissues from TCGA database.

Hierarchical clustering analysis indicated that 15 up- and down-regulated differentially expressed IncRNAs (A), microRNA (B) and mRNAs (C) were differentially expressed between HNSCC patients and healthy controls, Respectively. The red and the green shades represent the expression levels above and below the relative expression among all samples. 

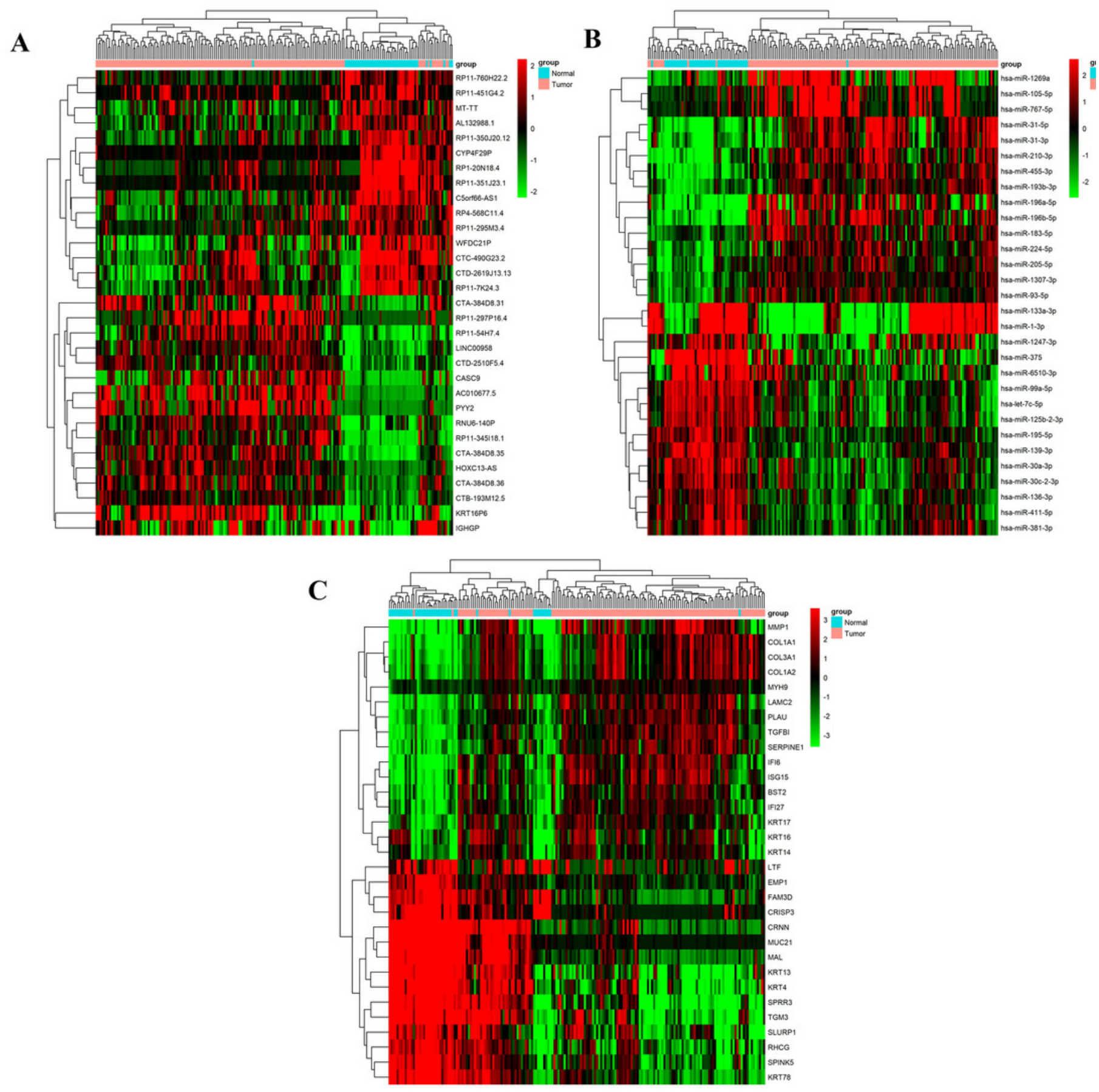


\section{Figure 3}

Figure 3 The 20 key Gene Ontology (GO) terms and KEGG pathway enrichment of differentially expressed intersection mRNAs for HNSCC groups versus adjacent normal controls.

A. The 20 Key gene ontology in up-regulated differentially expressed genes, the bar plot shows the enrichment scores of the significant enrichment $\mathrm{GO}$ terms. B. The 20 key gene ontology in down-regulated, differentially expressed genes, the bar plot shows the enrichment scores of the significant enrichment $\mathrm{GO}$ terms. C. The 20 key pathways in upregulated differentially expressed genes, the bar plot shows the enrichment scores of the significant enrichment pathways $\mathrm{D}$. The 20 key pathways in down-regulated differentially expressed genes.the bar plot shows the enrichment scores of the significant enrichment pathways. 
A

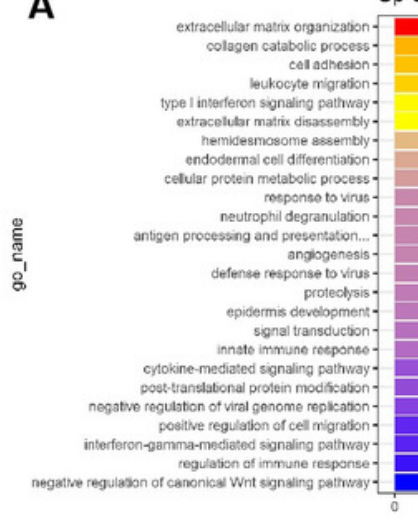

Up Gene Sig Top20 Gene Ontology

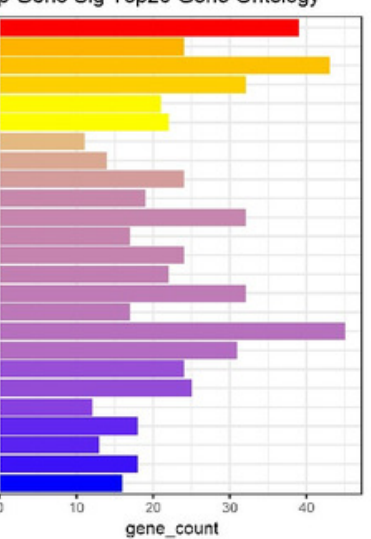

C

.

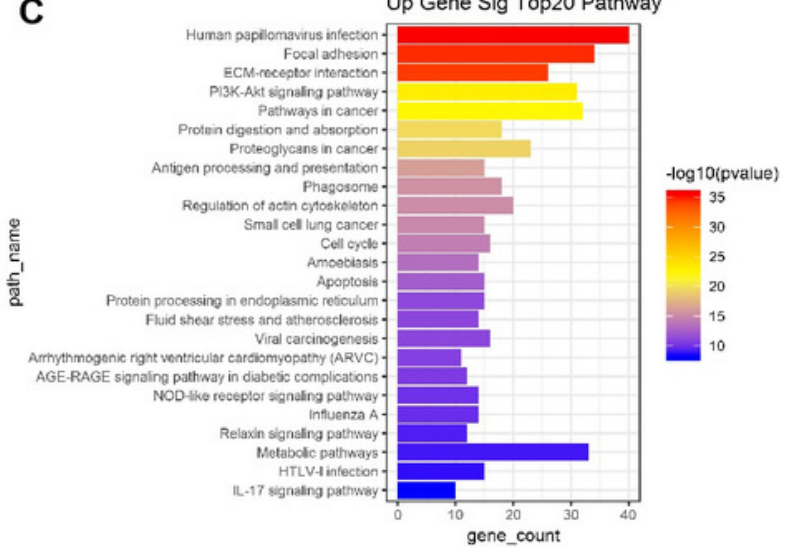

B
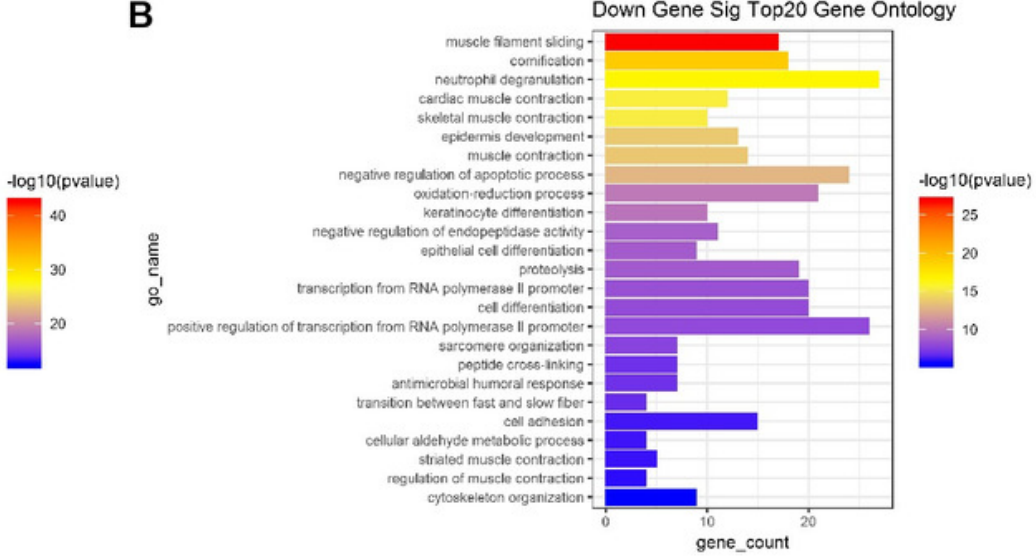

D

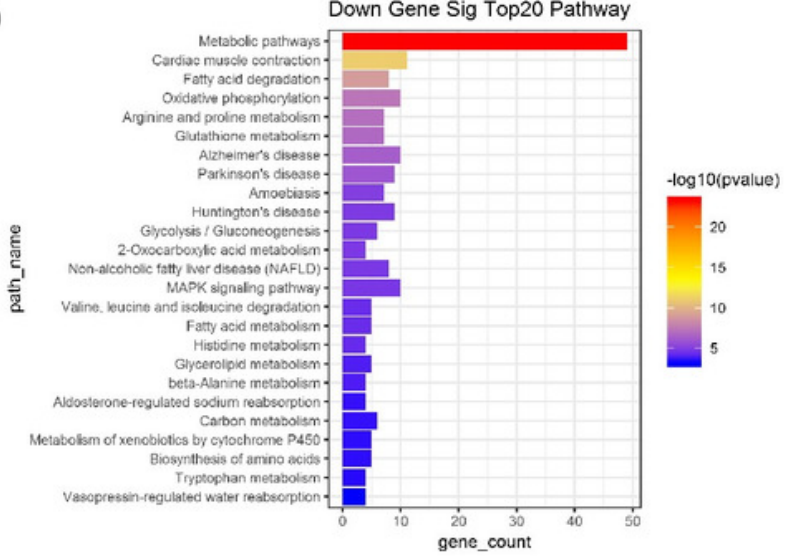




\section{Figure 4}

Figure 4 The ceRNA network of IncRNA-miRNA-mRNA in HNSCC.

The red represents the upregulated, and the green represents the downregulated. Diamonds represent miRNAs, balls represent mRNAs, and triangles represent IncRNAs. 


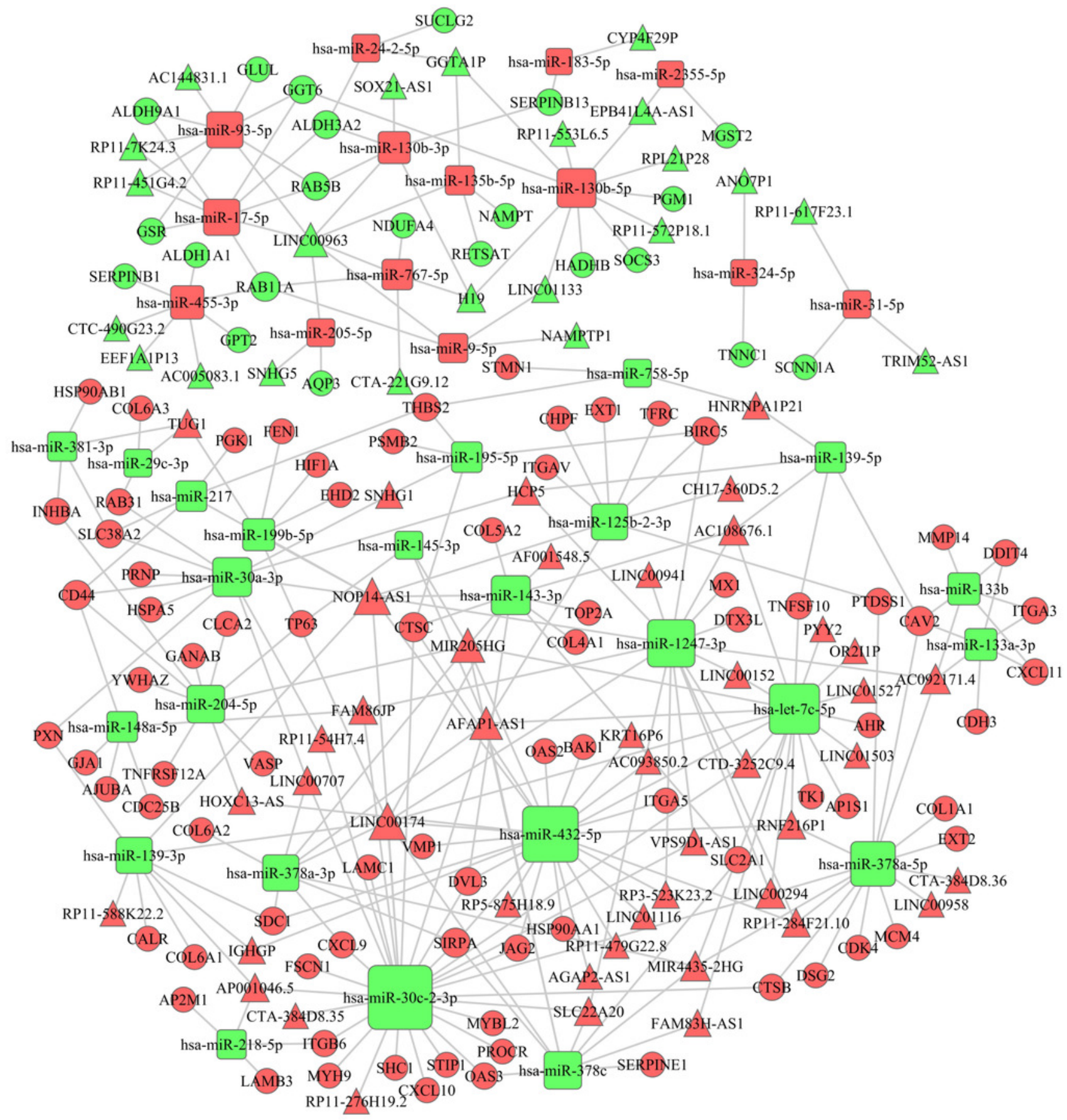


Figure 5

Figure 5 PPI network of 72 upregulated and 19 downregulated DEGs.

Nodes represent proteins and edges represent interactions between two proteins. Red nodes represent upregulated DEGs and green nodesrepresent downregulated DEGs.

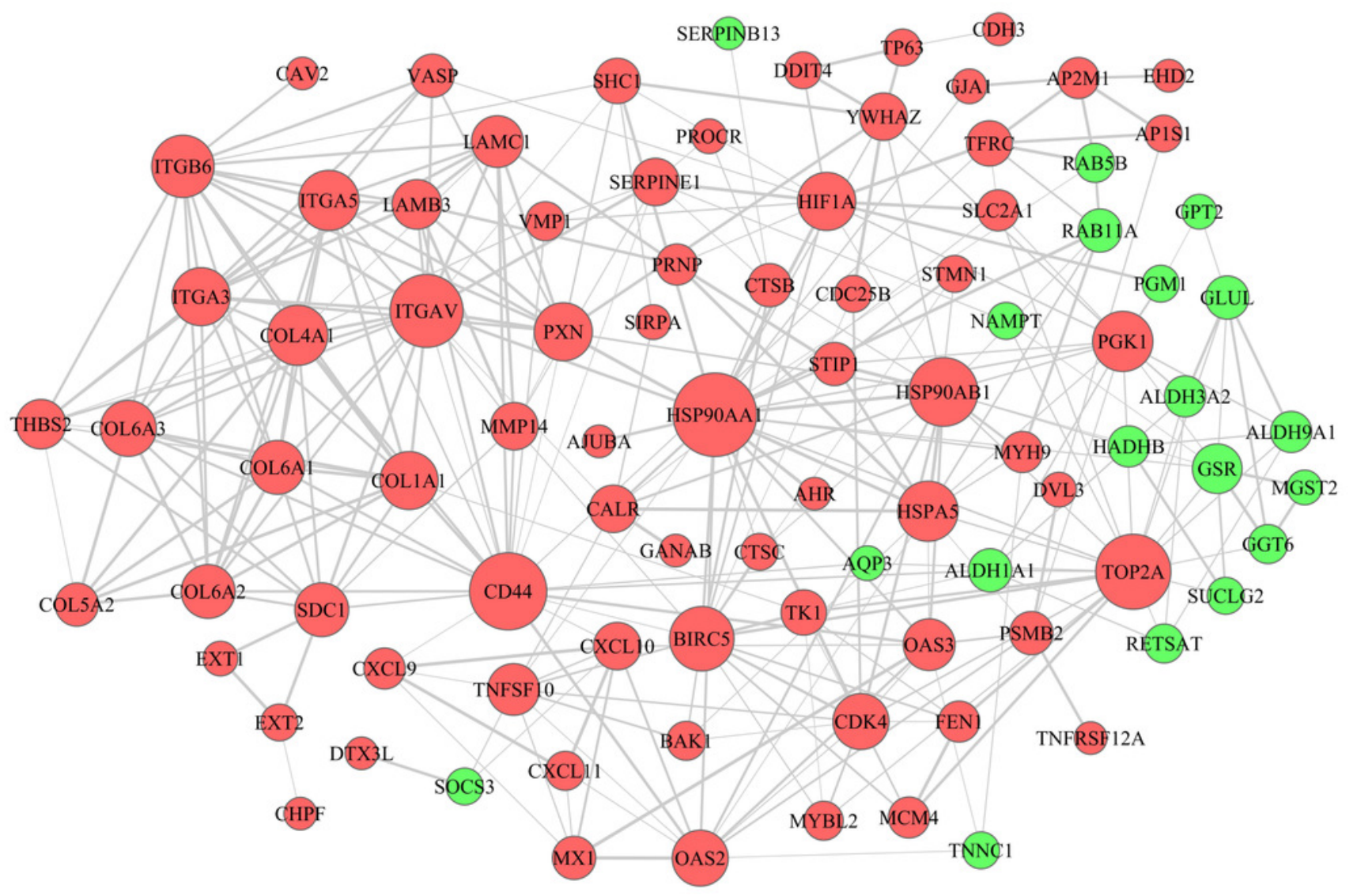




\section{Figure 6}

Figure 6 LINC00958 and HOXC13-AS expression levels in tumors and cells.

A-B. Enhanced expression of LINC00958 and HOXC13-AS expression levels in HNSCC tissues compared with adjacent normal tissues ( $n=44, T=141, p<0.0001)$. C-D. LINC00958 and HOXC13-AS expression levels in NPC cell lines were higher than that in NPEC2 Bmi-1 which was immortalized nasopharyngeal epithelial cells induced by Bmi-1. E-F. The ROC curve of LINC00958 and HOXC13-AS between HNSCC tissues and controls. 
A

B

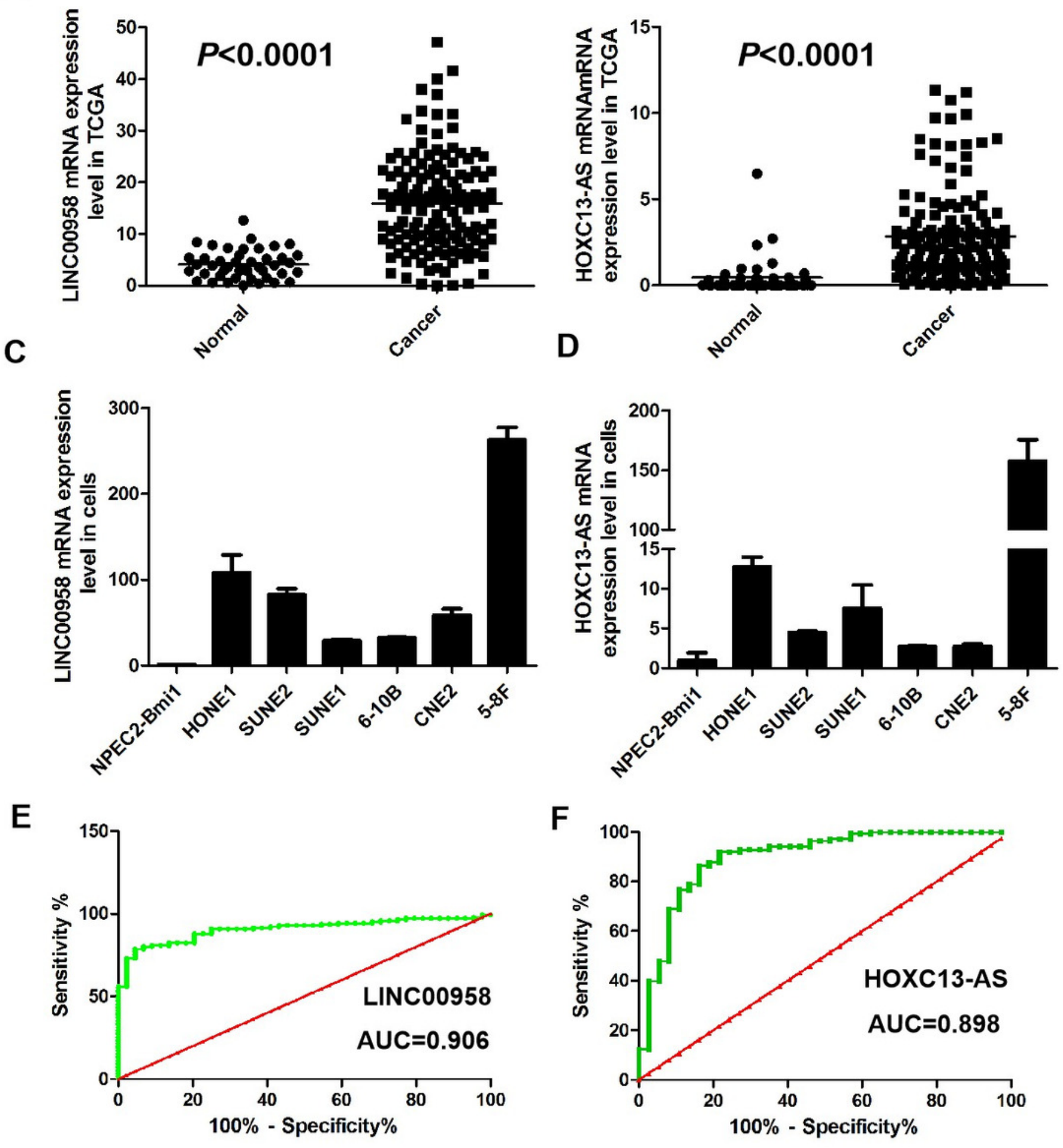




\section{Table $\mathbf{1}$ (on next page)}

The pathological stage information of head and neck squamous cell carcinoma tissues and adjacent normal tissues from TCGA database in this study 
1 Table 1 The pathological stage information of head and neck squamous cell carcinoma 2 tissues and adjacent normal tissues from TCGA database in this study.

3

4

5

6

7

8

9

10

11

12

13

14

15

16

17

\begin{tabular}{cccc}
\hline \multicolumn{2}{c}{ Adjacent normal tissues } & \multicolumn{2}{c}{ HNSCC tumor tissues } \\
\hline Stage & Number & Stage & Number \\
\hline NA & 1 & NA & 10 \\
stage I & 2 & stage I & 15 \\
stage II & 16 & stage II & 40 \\
stageIII & 8 & stageIII & 30 \\
stageIVa & 17 & stage IVa & 43 \\
& & stageIVb & 2 \\
& & stageIVc & 1 \\
\hline
\end{tabular}




\section{Table 2 (on next page)}

The origins information of head and neck squamous cell carcinoma tissues and adjacent normal tissues from TCGA database in this study 
1 Table 2 The origins information of head and neck squamous cell carcinoma tissues and 2 adjacent normal tissues from TCGA database in this study.

3

\begin{tabular}{ccc}
\hline & HNSCC & Normal \\
\hline Lip & 2 & 0 \\
Oropharynx & 2 & 0 \\
Base_of_Tongu & 3 & 2 \\
e & & \\
Hypopharynx & 3 & 0 \\
Hard_Palate & 4 & 0 \\
Alveolar_Ridge & 5 & 0 \\
Tonsil & 6 & 0 \\
Buccal_Mucosa & 10 & 0 \\
Floor_of_Mouth & 19 & 3 \\
Oral_Cavity & 21 & 14 \\
Larynx & 23 & 12 \\
Oral_Tongue & 43 & 13 \\
\hline
\end{tabular}

4

5

6

7

8

9

10

11

12

13 


\section{Table 3(on next page)}

The institutions information of head and neck squamous cell carcinoma tissues and adjacent normal tissues from TCGA database in this study 
1 Table 3 The institutions information of head and neck squamous cell carcinoma tissues 2 and adjacent normal tissues from TCGA database in this study.

3

\begin{tabular}{|c|c|}
\hline Institutions & Number \\
\hline Duke University & 1 \\
\hline Fox Chase & 1 \\
\hline ILSBio & 1 \\
\hline Medical College of Georgia & 1 \\
\hline Memorial Sloan Kettering Cancer Center & 1 \\
\hline Molecular Response & 1 \\
\hline St. University of Colorado Denver & 1 \\
\hline University Of Michigan & 1 \\
\hline ABS - IUPUI & 2 \\
\hline Barretos Cancer Hospital & 2 \\
\hline Fred Hutchinson & 2 \\
\hline Johns Hopkins & 2 \\
\hline Montefiore Medical Center & 2 \\
\hline University of Minnesota & 2 \\
\hline University of Schleswig-Holstein & 2 \\
\hline International Genomics Consortium & 3 \\
\hline University of Miami & 3 \\
\hline Emory University - Winship Cancer Inst. & 4 \\
\hline Asterand & 5 \\
\hline UNC & 6 \\
\hline Greater Poland Cancer Center & 10 \\
\hline Vanderbilt University & 13 \\
\hline University Health Network, Toronto & 19 \\
\hline University of Pittsburgh & 21 \\
\hline MD Anderson Cancer Center & 79 \\
\hline
\end{tabular}

